\title{
Susceptibility of Neonatal Lipoproteins to Oxidative Stress
}

\author{
TOHRU OGIHARA, MAKOTO KITAGAWA, MASAYUKI MIKI, HIROSHI TAMAI, \\ HIROSHI YASUDA, RYOZO OKAMOTO, AND MAKOTO MINO \\ Department of Pediatrics, Osaka Medical College, Takatsuki City, Osaka, 569, Japan
}

\begin{abstract}
We compared peroxidizability of neonatal and adult lipoproteins exposed to oxidative stress initiated by an azo-compound. Oxygen uptake showed a two-phase pattern, with slow oxygen uptake in the first phase and faster uptake in the second phase. During the first phase, tocopherol was consumed progressively, inhibiting lipid peroxidation by scavenging peroxy radicals. After the tocopherol concentration fell below a critical level, extensive propagation of chain oxidative reactions produced the rapid oxygen uptake in the second phase. In the first phase of neonatal lipoprotein oxidation, a faster oxygen uptake was observed and the length of this phase was shorter than in adults. The oxygen uptake rate in the second phase was smaller in the neonatal lipoproteins. In other words, neonatal lipoproteins were more susceptible to oxidative stress than were adult ones when tocopherol remained in lipoproteins, but after tocopherol depletion the reverse was true. These results were consistent with the finding that in neonatal lipoproteins the tocopherol/lipid ratio was significantly lower (length of the inhibitory phase was closely correlated to the lipoprotein tocopherol content). In addition, the average number of active bisallylic hydrogen atoms, which are considered to determine the relative susceptibility of polyunsaturated fatty acids to oxidation, was significantly smaller (oxygen uptake rate in the second phase was closely correlated to the active bisallylic hydrogen number) in neonatal lipoproteins, and the ratio of active bisallylic hydrogen to tocopherol content (which closely correlated to oxygen uptake rate in the first phase) was also significantly lower compared with the adult ratio. Under physiologic conditions, an intensive oxidation of lipoproteins sufficient to cause lipoprotein tocopherol to fall below critical levels is unlikely to occur. Therefore, it seems reasonable to suggest that neonatal lipoproteins are more susceptible to oxidative stress than are adult lipoproteins. (Pediatr Res 29: 39-45, 1991)
\end{abstract}

\section{Abbreviations}

PUFA, polyunsaturated fatty acid

AAPH, 2,2'-azobis (2-amidinopropane) dihydrochloride

active $H$, active bisallylic hydrogen

$T_{\text {inh }}$, length of the inhibitory phase of oxygen uptake (first phase) (s)

$R_{\text {inh }}$, oxygen uptake rate in the inhibitory phase (first phase) (nM/s)

Received March 19, 1990; accepted September 7, 1990.

Correspondence and reprint requests: Tohru Ogihara, M.D., Department of Pediatrics, Osaka Medical College, 2-7, Daigakucho, Takatsuki City, Osaka, 569, Japan.

Supported by a Grant-in-Aid (61480225) for Scientific Research from the Ministry of Education, Science, and Culture, Japan.
$\mathbf{R}_{\text {prop }}$ oxygen uptake rate in the propagation phase (second phase) (nM/s)

VE, vitamin E

Recently, the modification of LDL by free radical oxidation has received widespread attention in connection with the pathogenesis of atherosclerosis $(1,2)$. It is also known that oxidized LDL shows cytotoxicity in vitro to certain cells (3). Moreover, a wide range of highly cytotoxic lipid peroxidative products, such as toxic aldehydes, can be generated from oxidized lipoproteins (4). Therefore, it is possible that lipoproteins modified by oxidation may contribute to various disorders besides atherosclerosis. In the neonatal period, plasma lipoproteins may be frequently exposed to oxidative stress, due to conditions such as respiratory distress, infections, or hemorrhage. The peroxidation of lipoproteins may then contribute to any pathologic sequelae occurring in neonates with the above disorders.

In circulation, free radicals formed in the aqueous region are scavenged extensively by hydrophilic antioxidants, such as ascorbic acid, uric acid, bilirubin, and others (5). However, when oxidative ability of these radicals exceeds the protective capacity of the hydrophilic scavengers and affects lipoproteins, or when the radicals are generated initially within the lipoproteins themselves, tocopherol incorporated into lipoproteins may act as the main antioxidant to protect against free radical attack.

We have previously shown that the distribution of tocopherol among lipoproteins is directly related to the total lipid content of the lipoprotein fraction (6), and that the tocopherol concentrations expressed in terms of the total lipids (tocopherol/lipid ratio) in each lipoprotein fraction are therefore similar to the ratio in whole plasma. The plasma tocopherol/lipid ratio, which has been proposed as the most reliable index for the assessment of VE status $(7,8)$, has been reported to be at an acceptable level (more than 0.8 ) in neonates (9). This suggests that neonates are not in a VE-deficient state. Moreover, this hypothesis is supported by the finding that erythrocyte tocopherol levels in neonates are comparable to the levels in adults $(10,11)$ because erythrocyte tocopherol levels are also considered to be an index for the assessment of VE status $(12,13)$. Despite this apparent sufficiency of VE in neonatal erythrocytes, their erythrocytes are known to be highly susceptible to oxidative stress, as has been shown by the report of their increased susceptibility to hydrogen peroxide hemolysis (14). Also, we have recently reported that neonatal erythrocyte membranes are highly peroxidizable in response to oxidative stress when compared with adult membranes (15).

Although the plasma tocopherol/lipid ratio is above the critical level for VE deficiency in neonates, it is still significantly lower in comparison with that in adults. This lower tocopherol/lipid ratio suggests that neonatal lipoproteins may be more susceptible to oxidative stress than adult lipoproteins. In this study, we 
Table 1. Tocopherol/lipid ratio in plasma and lipoprotein fractions*

\begin{tabular}{|c|c|c|c|c|c|c|}
\hline & \multicolumn{3}{|c|}{ Neonates } & \multicolumn{3}{|c|}{ Adults } \\
\hline & $\alpha-$ Toct & $\gamma$-Toc & Total Toc & $\alpha$-Toc & $\gamma$-Toc & Total Toc \\
\hline Plasma & $1.32 \pm 0.14$ & $0.09 \pm 0.03$ & $1.41 \pm 0.14$ & $1.68 \pm 0.23$ & $0.31 \pm 0.09$ & $1.99 \pm 0.23$ \\
\hline VLDL & $1.26 \pm 0.24$ & $0.06 \pm 0.02$ & $1.32 \pm 0.25$ & $1.83 \pm 0.32$ & $0.31 \pm 0.11$ & $2.14 \pm 0.37$ \\
\hline LDL & $1.31 \pm 0.21$ & $0.07 \pm 0.04$ & $1.47 \pm 0.37$ & $1.82 \pm 0.38$ & $0.22 \pm 0.08$ & $1.96 \pm 0.42$ \\
\hline HDL & $1.38 \pm 0.22$ & $0.12 \pm 0.04$ & $1.45 \pm 0.30$ & $1.86 \pm 0.53$ & $0.29 \pm 0.11$ & $2.02 \pm 0.46$ \\
\hline
\end{tabular}

* Values are the mean \pm SD of 10 neonatal and 10 adult lipoprotein samples ( $\mu \mathrm{g} / \mathrm{mg}$ total lipids). $p<0.001$ vs adults for all values.

$\uparrow$ Toc, tocopherol.

Table 2. Fatty acid compositions and active $H$ number in neonatal and adult lipoproteins*

\begin{tabular}{|c|c|c|c|c|}
\hline & \multicolumn{2}{|c|}{ LDL } & \multicolumn{2}{|c|}{ HDL } \\
\hline & Neonates & Adults & Neonates & Adults \\
\hline C 14:0 & $1.6 \pm 0.2$ & $1.2 \pm 0.3$ & $0.9 \pm 0.2 \dagger$ & $0.8 \pm 0.3$ \\
\hline C 16:0 & $39.1 \pm 3.1$ & $24.5 \pm 2.3$ & $42.5 \pm 2.6$ & $30.1 \pm 2.2$ \\
\hline C 18:0 & $5.4 \pm 0.9$ & $3.2 \pm 0.4$ & $10.3 \pm 1.9$ & $7.2 \pm 1.5$ \\
\hline C 18:1 & $22.7 \pm 3.6$ & $14.0 \pm 1.5$ & $15.2 \pm 2.1$ & $11.8 \pm 1.4$ \\
\hline C $18: 2$ & $14.7 \pm 3.3$ & $46.1 \pm 4.4$ & $11.5 \pm 2.1$ & $35.3 \pm 7.0$ \\
\hline C $18: 3$ & $0.4 \pm 0.4 \dagger$ & $0.5 \pm 0.2$ & $0.2 \pm 0.1 \dagger$ & $0.3 \pm 0.1$ \\
\hline C $20: 3$ & $1.5 \pm 0.2$ & $0.5 \pm 0.1$ & $2.5 \pm 0.3$ & $0.8 \pm 0.1$ \\
\hline C $20: 4$ & $8.2 \pm 1.9$ & $5.0 \pm 0.7$ & $11.1 \pm 1.7$ & $6.5 \pm 1.4$ \\
\hline C 20:5 & $0.5 \pm 0.2$ & $1.5 \pm 0.7$ & $0.4 \pm 0.2$ & $2.0 \pm 1.5$ \\
\hline C 22:0 & $0.2 \pm 0.1 \dagger$ & $0.2 \pm 0.1$ & $0.4 \pm 0.1$ & $0.2 \pm 0.1$ \\
\hline C 22:5 & $0.2 \pm 0.1 \dagger$ & $0.2 \pm 0.1$ & $0.2 \pm 0.1 \dagger$ & $0.3 \pm 0.2$ \\
\hline C 22:6 & $3.3 \pm 1.1$ & $1.9 \pm 0.6$ & $2.9 \pm 0.7$ & $2.6 \pm 0.9$ \\
\hline Active $\mathrm{H}$ & $1.27 \pm 0.29$ & $1.62 \pm 0.16$ & $1.36 \pm 0.20$ & $1.61 \pm 0.25$ \\
\hline
\end{tabular}

* Values are the mean \pm SD (mol \%) of 20 neonatal lipoprotein samples and 16 adult lipoprotein samples. Average number of bisallylic hydrogens (active $\mathrm{H}$ ) in one molecule of fatty acid calculated from the fatty acid compositions. $p<0.001 v s$ adults for all values except those with footnotes.

$\dagger$ Not significantly different $v s$ adults.

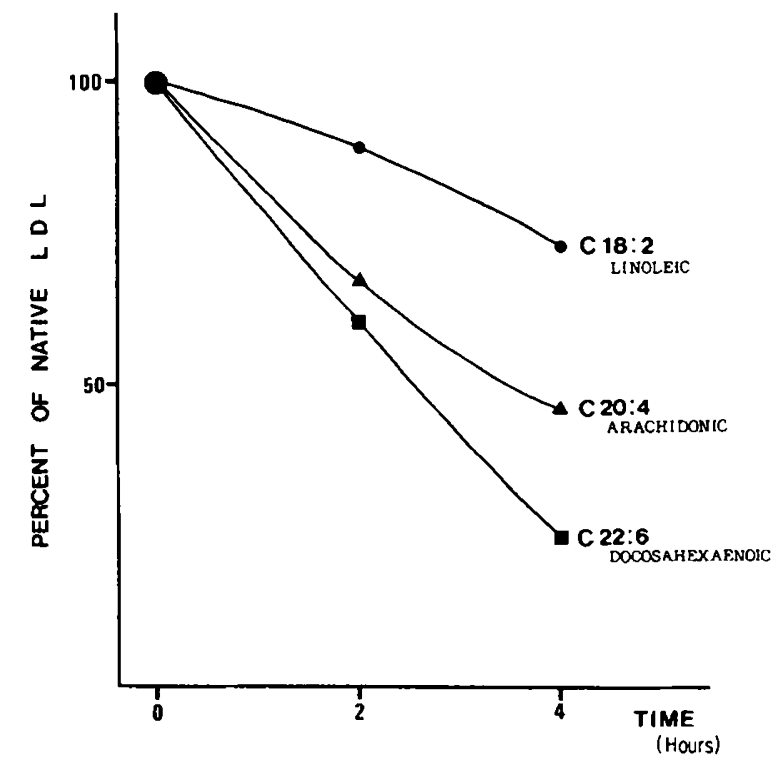

Fig. 1. The decrease of PUFA during oxidation of adult LDL. Dialyzed LDL (1 mg total lipids/mL) was incubated with $20 \mathrm{mM}$ AAPH in isotonic phosphate buffer containing $1 \mathrm{mM}$ EDTA ( $\mathrm{pH} \mathrm{7.4)}$ at $37^{\circ} \mathrm{C}$ with constant stirring by a magnetic stirrer. At the indicated time points, the fatty acids were analyzed by gas-liquid chromatograph. Plots are the mean of three experiments. Results are expressed as percent change from the native $\mathrm{LDL}$. investigated the peroxidizability of neonatal lipoproteins under oxidative stress in comparison with adult lipoproteins, using an azo-compound as a radical initiator.

\section{MATERIALS AND METHODS}

Materials. As neonatal blood, cord blood was obtained in heparin-treated tubes through the umbilical vein from separated placentas immediately after cord clamping. Healthy, normal birth, full-term infants with Apgar scores of greater than 9 at 5 min were selected. Their birth weights and gestational ages averaged $3100 \mathrm{~g}$ (ranging from the 10 th to the 90 th percentile) and $39 \mathrm{wk}$ (ranging from 38 to $41 \mathrm{wks}$ ), respectively. Their mothers had no problems during pregnancies and deliveries. As adult samples, heparinized blood was drawn from healthy adult male volunteers (aged from 20 to $33 \mathrm{y}$ ) after overnight fasting. To obtain tocopherol-rich lipoproteins, some of the volunteers were orally given $\mathrm{d}$ - $\alpha$-tocopherol $600 \mathrm{mg}$ daily for $5 \mathrm{~d}$. The study protocol was approved by the ethics committee of the college hospital, and the studies were performed after informed consent.

Lipoprotein separation. The red blood cells were removed by $1500 \times g$ centrifugation for $10 \mathrm{~min}$ within $30 \mathrm{~min}$ after blood sampling. Plasma lipoproteins were fractionated by ultracentrifugation according to the method of Hatch and Lees (16), within $6 \mathrm{~h}$ after isolation of the plasma. The plasma was centrifuged sequentially with adjustment of density to $1.006,1.063$, and 1.21 by $\mathrm{NaBr}$ at $4^{\circ} \mathrm{C}$, using a Hitachi $65 \mathrm{P}-7$ ultracentrifuge with a type RP-65 rotor (Hitachi Koki Co., Ltd., Tokyo, Japan). The 1.006 $<$ density $<1.063$ fractions were expressed as LDL and the 1.063 $<$ density $<1.21$ as HDL in this study. The lipoproteins obtained were then dialyzed for $24 \mathrm{~h}$ at $4^{\circ} \mathrm{C}$ against an isotonic solution containing $10 \mathrm{mM}$ phosphate buffer, $125 \mathrm{mM} \mathrm{NaCl}$, and $1 \mathrm{mM}$ EDTA (pH 7.4).

Oxidation procedures. Using a slight modification of the method of Yamamoto et al. (17), we took an azo-compound, $\mathrm{AAPH}$, as a water-soluble radical initiator. AAPH produces initiating radicals at a constant rate by unimolecular thermal decomposition and the rate of free radicals can be easily controlled by adjusting the concentration of the initiator. This is essential for quantitative kinetic studies.

The dialyzed lipoproteins were suspended in the isotonic phosphate buffer described above to give $1 \mathrm{mg}$ of total lipids $/ \mathrm{mL}$ (estimated by the addition of three major lipids, i.e. total cholesterol, phospholipids, and triglycerides) and $20 \mathrm{mM} \mathrm{AAPH}$ in the final reaction solution. The reaction mixture was then poured into a reaction vessel and kept at $37^{\circ} \mathrm{C}$ to start the oxidation of lipoproteins, with constant stirring by a magnetic stirrer. The rate of lipoprotein oxidation was followed by the decrease of oxygen concentration in the reaction mixture due to oxygen consumption measured using an Oxygraph model 5/6 (Gilson Medical Electronics Inc., Middleton, WI).

Determination of fatty acid composition of lipoproteins. Fatty acid methyl esters were prepared by the one-step method, eliminating the extraction step using 2,2'-dimethoxypropane, as described by Shimasaki et al. (18). The fatty acid composition of the acetone solution of the extract was analyzed by a gas-liquid chromatograph with a flame ionization detector (Shimadzu GC- 


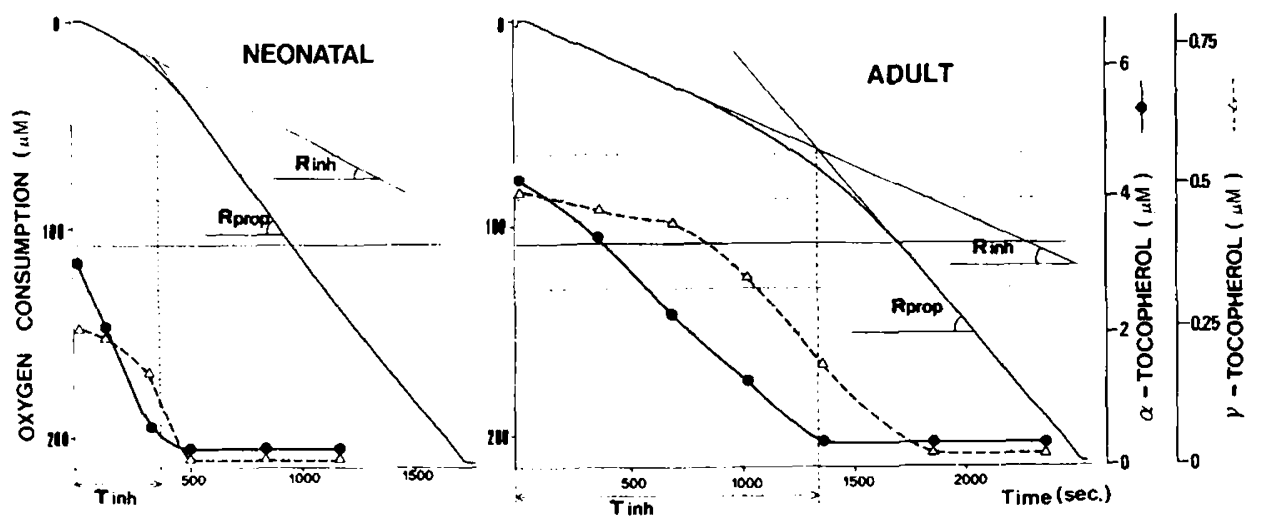

Fig. 2. Examples of oxygen consumption pattern during oxidation of neonatal and adult HDL. Dialyzed HDL ( $1 \mathrm{mg}$ total lipids/mL) was incubated with $20 \mathrm{mM} \mathrm{AAPH}$ in isotonic phosphate buffer containing $1 \mathrm{mM} \mathrm{EDTA} \mathrm{(pH} \mathrm{7.4)} \mathrm{at} 37^{\circ} \mathrm{C}$ with constant stirring by a magnetic stirrer. Oxygen concentration in the reaction mixture was monitored continuously. At the indicated time points, $\alpha-(\bullet)$ and $\gamma(\Delta)$-tocopherol concentrations were measured. Each point represents a single observation.
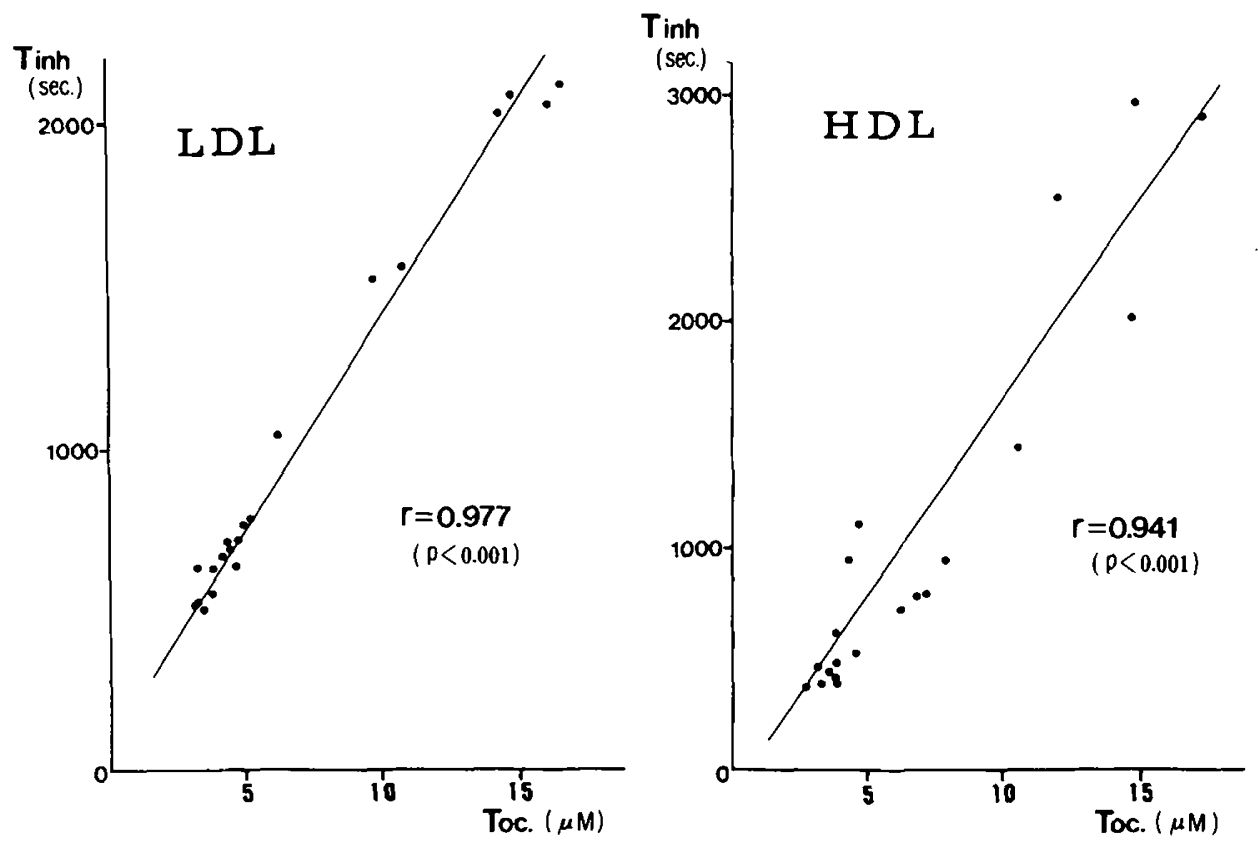

Fig. 3. Correlation between $T_{\text {inh }}$ and total tocopherol content during oxidation of LDL and HDL. Oxidation procedure was as described in Materials and Methods and the legend for Figure 2. Oxygen concentration in the reaction mixture was monitored continuously and $\Upsilon_{i n h}$ was measured from the oxygen consumption pattern as, for example, shown in Figure 2. Ten neonatal samples and 10 adult samples, containing six tocopherol-rich samples obtained from adult volunteers who were receiving $\mathrm{d}$ - $\alpha$-tocopherol, $600 \mathrm{mg}$ daily for $5 \mathrm{~d}$, were examined. Each point represents an individual data measurement.

8A, Shimadzu Corporation, Kyoto, Japan), as previously described (19).

Assays for tocopherol and major lipids. Levels of total cholesterol, phospholipids, and triglycerides were measured by the enzymatic methods of Allain et al. (20). Takayama et al. (21), and Bucolo et al. (22), respectively, using Wako Kits (Wako Chemicals, Tokyo, Japan). Alpha- and $\gamma$-tocopherol levels in lipoprotein fractions were analyzed by Ishibashi's (23) modification, using electrochemical detection, as described in our previous paper (24). The instruments used in the assay for tocopherol were as follows: high-performance liquid chromatograph, IRIKA P-530 (IRIKA Instruments Inc., Kyoto, Japan); column, IRIKA RP-18 (C:18), $4 \times 250 \mathrm{~mm}$ column; eluent, methanol/ water $/ \mathrm{NaClO}_{4}=1000 / 2 / 7(\mathrm{vol} / \mathrm{vol} / \mathrm{wt}) ;$ flow rate, $10 \mathrm{~mL} / \mathrm{min}$; and detector, IRIKA Amperometric E-520.

Statistical analysis. All results are expressed as mean \pm SD Statistical significance was assessed using the unpaired $t$ test. Linear regression analysis was performed using the least squares nethod.

\section{RESULTS}

Lipoprotein tocopherol/lipid ratios. Table 1 shows the tocopherol/lipid ratio in whole plasma and the individual lipoproteins in neonates and healthy adults. The ratios calculated for $\alpha-, \gamma-$, and total tocopherol were similar in plasma and each lipoprotein fraction. The values in neonates were significantly lower than those in adults in plasma and each lipoprotein fraction, although the ratios remained above the critical level of 0.8 , which implies VE deficiency.

Fatty acid composition of lipoproteins. Table 2 shows the fatty acid composition of lipoprotein lipids in both groups. Neonatal lipoproteins in both LDL and HDL were rich in PUFA, particularly eichosatrienoic (C20:3), arachidonic (C20:4), and docosahexaenoic acid (C22:6), when compared with the adult lipoproteins. Among the PUFA, only the percentage of linoleic acid (C1 8:2) was markedly lower in neonates. The PUFA composition of LDL was similar to that of HDL, although LDL showed a somewhat greater percentage of linoleic acid and a lesser percentage of arachidonic acid than HDL in both groups. 

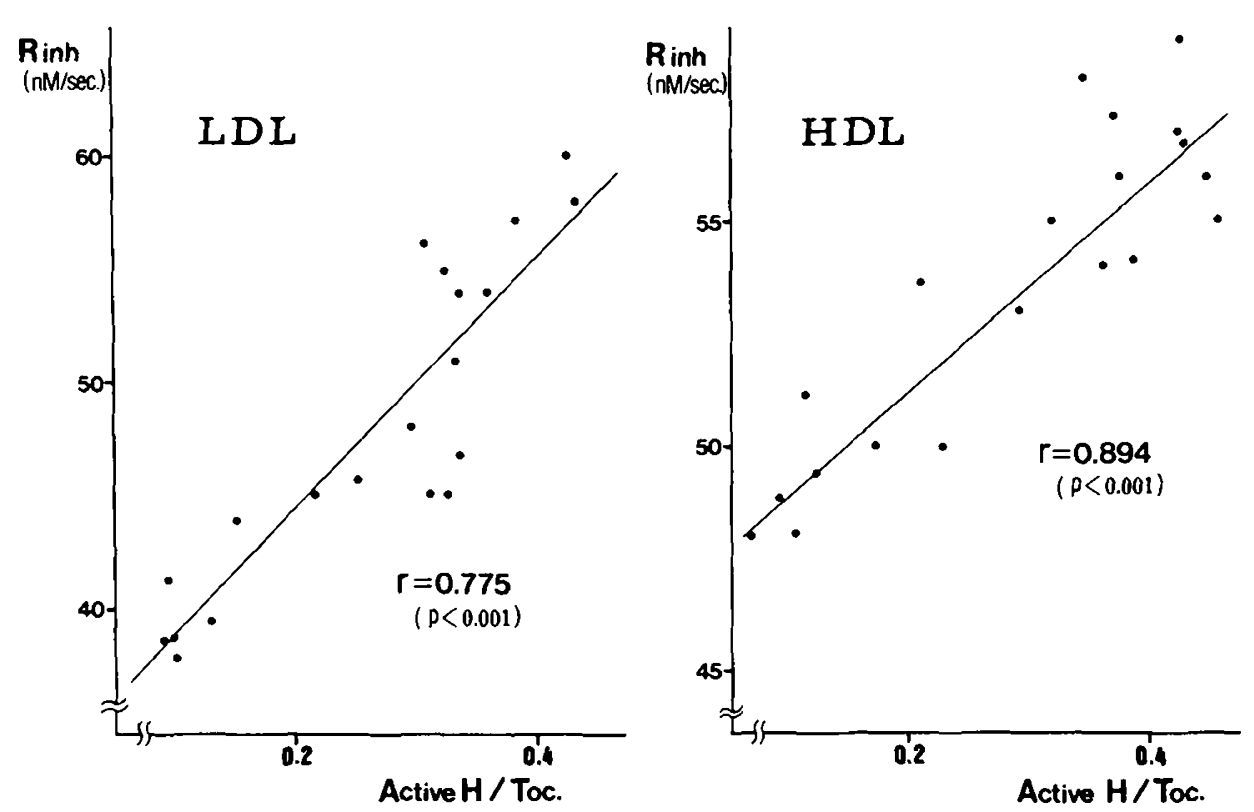

Fig. 4. Correlation between $R_{\text {inh }}$ and active $H /$ tocopherol ratio during oxidation of $L D L$ and $H D L$. Oxidation procedure was as described in Materials and Methods and the legend for Figure 2. $R_{\text {inh }}$ and active $H /$ tocopherol ratio were measured from the same samples and experiments shown in Figure 3.
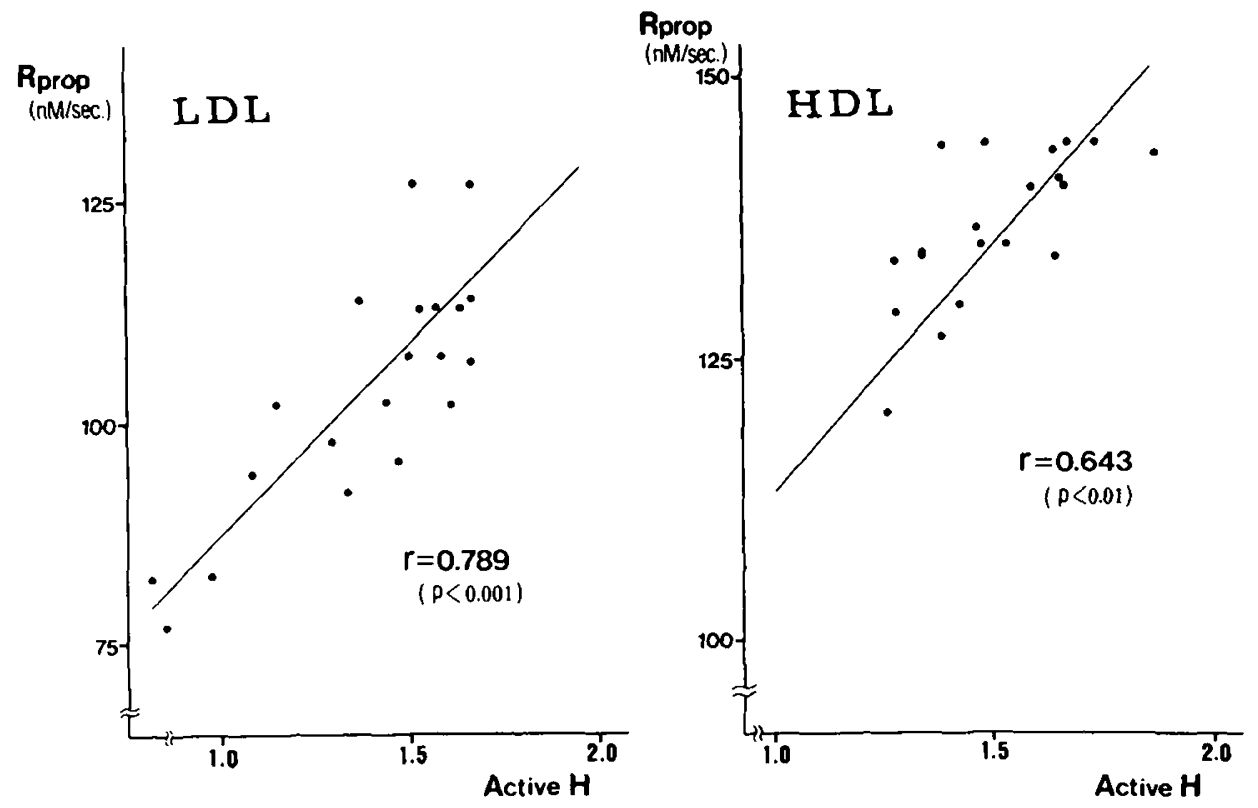

Fig. 5. Correlation between $R_{\text {prop }}$ and active $H$ during oxidation of LDL and HDL. Oxidation procedure was as described in Materials and Methods and the legend for Figure 2. $\mathrm{R}_{\text {prop }}$ and active $\mathrm{H}$ were measured from the same samples and experiments shown in Figure 3.

Table 3. $T_{\text {inh }}$ and total tocopherol content of neonatal and adult

\begin{tabular}{lccccc}
\hline & \multicolumn{2}{c}{ lipoproteins* $^{*}$} & & \multicolumn{2}{c}{ HDL } \\
\cline { 2 - 3 } \cline { 5 - 6 } & Neonates & Adults & & Neonates & Adults \\
\hline $\mathrm{T}_{\text {inh }}(\mathrm{s})$ & $595 \pm 140 \dagger$ & $854 \pm 271$ & & $402 \pm 89 \ddagger$ & $924 \pm 384$ \\
Total Toc & $3.42 \pm 0.86 \ddagger$ & $4.57 \pm 0.98$ & $3.37 \pm 0.30 \ddagger$ & $4.71 \pm 0.42$
\end{tabular}
$(\mu \mathrm{M}) \S$

* Values are the mean \pm SD of 20 neonatal samples and 20 adult samples.

$\dagger p<0.01$ vs adults.

$\ddagger p<0.001$ vs adults.

$\S$ Toc, tocopherol.
Because it is generally accepted that the hydrogen atoms attached to the carbon atoms located between the double bonds of PUFA are preferentially abstracted by free radicals, the relative susceptibility of PUFA to oxidation can be determined by the number of these hydrogens (25). In this report, these hydrogen atoms are designated as active $H$. The average active $H$ number in one molecule of fatty acid was calculated for each fatty acid by doubling a number one less than the number of double bonds. As shown in Figure 1, when LDL was oxidized with AAPH, PUFA with higher active $\mathrm{H}$ numbers were decomposed faster than those with lower active $\mathrm{H}$ numbers. For example, arachidonic acid decreased faster than linoleic acid because the former possesses six active $\mathrm{H}$ in one molecule, whereas the latter has only two.

The average active $\mathrm{H}$ number in neonatal lipoproteins was found to be significantly smaller than that in adult lipoproteins 
Table 4. $R_{\text {inh }}$ and active H/tocopherol ratio of neonatal and adult lipoproteins*

\begin{tabular}{lccccc}
\hline & \multicolumn{2}{c}{ LDL } & & \multicolumn{2}{c}{ HDL } \\
\cline { 2 - 4 } \cline { 5 - 6 } & Neonates & Adults & & Neonates & Adults \\
\hline$R_{\text {inh }}(\mathrm{nM} / \mathrm{s})$ & $50 \pm 5 \dagger$ & $45 \pm 3$ & & $57 \pm 5 \ddagger$ & $50 \pm 4$ \\
Active-H/Toc (average no. $/ \mu \mathrm{M}) \S$ & $0.37 \pm 0.07 \|$ & $0.32 \pm 0.05$ & & $0.42 \pm 0.06 \ddagger$ & $0.30 \pm 0.05$ \\
\hline
\end{tabular}

* Values are the mean \pm SD of 20 neonatal samples and 20 adult samples.

$\dagger p<0.01$ vs adults.

$\ddagger p<0.001$ vs adults.

$\S$ Toc, tocopherol.

$\| p<0.02$ vs adults.

Table 5. $R_{\text {prop }}$ and active $H$ number of neonatal and adult lipoproteins*

\begin{tabular}{lcccc}
\hline & \multicolumn{2}{c}{ LDL } & \multicolumn{2}{c}{ HDL } \\
\cline { 2 - 5 } & Neonates & Adults & Neonates & Adults \\
\hline$R_{\text {prop }}(n M / s)$ & $95 \pm 12 \dagger$ & $122 \pm 15$ & $129 \pm 15 \ddagger$ & $138 \pm 5$ \\
$\begin{array}{l}\text { Active H (average no. of one mole- } \\
\text { cule FFA) }\end{array}$ & $1.27 \pm 0.29 \S$ & $1.62 \pm 0.16$ & $1.36 \pm 0.20 \S$ & $1.61 \pm 0.25$ \\
\hline
\end{tabular}

* Values are the mean \pm SD of 20 neonatal samples and 20 adult samples.

$\dagger p<0.001$ vs adults.

$\ddagger p<0.05$ vs adults.

$\S p<0.01$ vs adults.

(Table 2), reflecting the marked difference in linoleic acid content between these two groups, despite the higher content of other PUFA in neonatal lipoproteins.

Oxygen consumption of lipoproteins during reaction with $A A P H$. The oxidation of lipids in lipoproteins induced by AAPH proceeded by the following mechanism $(19,25,26)$, where $\mathrm{AOO}^{\circ}=$ initiating radical, $\mathrm{LH}=$ lipid, $\mathrm{L}^{\circ}=$ lipid radical, $\mathrm{LOO}^{\circ}$ $=$ lipid peroxy radical, $\mathrm{LOOH}=$ hydroperoxide, $\mathrm{IH}=$ antioxidant, and I = antioxidant radical.

Chain initiation

$$
\begin{aligned}
& \mathrm{AAPH}+2 \mathrm{O}_{2} \rightarrow 2 \mathrm{AOO}+\mathrm{N}_{2} \\
& \mathrm{AOO}+\mathrm{LH} \rightarrow \mathrm{AOOH}+\mathrm{LOO}
\end{aligned}
$$

Propagation

$$
\begin{gathered}
\mathrm{LOO}^{\circ}+\mathrm{LH} \rightarrow \mathrm{LOOH}+\mathrm{L}^{\circ} \\
\mathrm{L}^{\prime}+\mathrm{O}_{2} \rightarrow \mathrm{LOO}^{\circ}
\end{gathered}
$$

Competitive inhibition by antioxidant

$$
\mathrm{LOO}^{\circ}+\mathrm{IH} \rightarrow \mathrm{LOOH}+\mathrm{I}^{\circ}
$$

Termination

$$
2 \mathrm{LOO}^{\circ} \rightarrow \text { nonradical products }
$$

In this scheme, LH means PUFA in lipoprotein oxidation, because these are preferentially attacked by free radicals during the oxidation of lipids. An initiating radical (AOO) attacks PUFA in lipoproteins and generates lipid peroxy radical (LOO') (reaction 2). LOO' attacks other lipid molecules to produce hydroperoxide $(\mathrm{LOOH})$ and new lipid radical (L) (reaction 3 ). Then $\mathrm{L}^{\prime}$ reacts with oxygen to generate further $\mathrm{LOO}^{\prime}$ (reaction 4). As reactions 3 and 4 take place repeatedly, the oxidation proceeds by a free radical chain mechanism. By competing with LH in reacting with LOO, antioxidant $(\mathrm{IH})$ can inhibit this chain propagation of lipid peroxidation (reaction 5). In lipoproteins, only tocopherol may act as a chain-breaking, fat-soluble antioxidant, except for a small amount of $\beta$-carotene and ubiquinol (Niki E, personal communication).

Needless to say, AAPH is not a physiologic substance. No matter what the initiator may be, as long as the free radical chain mechanism of lipid peroxidation occurs, the initiator should not make any difference to the consequences. Therefore, this method should be effective when comparing the peroxidizability of neonatal and adult lipoproteins.

When lipoproteins were reacted with AAPH, the oxygen consumption pattern shared two clearly distinguishable phases (Fig. 2). Initially, oxygen was consumed at a slow rate (first phase), but subsequently rapid oxygen consumption occurred (second phase). Simultaneous analysis of tocopherol concentration revealed that tocopherol was consumed progressively during the first phase. Initially, only $\alpha$-tocopherol disappeared linearly and $\gamma$-tocopherol levels remained almost unchanged, and then $\gamma$ tocopherol was consumed after $\alpha$-tocopherol had been consumed to some extent. After tocopherol concentrations fell below critical levels, rapid oxygen consumption was observed. In this report, the oxygen consumption rate in the first slower phase is denoted by $R_{\text {inh }}$, and that in the faster second phase by $R_{\text {prop }}$, and both are expressed as $\mathrm{nM}$ of oxygen consumption per $\mathrm{s}$. The length of the first phase is referred to as $T_{\text {inh }}$, and expressed in $\mathrm{s}$.

We examined the correlations between tocopherol content, active $\mathrm{H}$ number, and oxygen consumption pattern on the basis of the experiments concerning the neonatal, adult, and tocopherol-rich adult lipoproteins. $T_{\text {inh }}$ was closely correlated to the tocopherol content of lipoproteins (Fig. 3). $R_{\text {inh }}$ was correlated to the ratio of active $\mathrm{H}$ to tocopherol content (Fig. 4), and a close correlation between $R_{\text {prop }}$ and the active $H$ number was observed (Fig. 5).

The changes in these parameters in neonatal and adult lipoproteins exposed to AAPH are summarized in Tables 3, 4, and 5. $T_{\text {inh }}$ was markedly shorter for neonatal lipoproteins (Table 3 ). Also, $R_{\text {inh }}$ was greater for neonatal lipoproteins (Table 4), whereas $R_{\text {prop }}$ was greater for adult lipoproteins (Table 5).

\section{DISCUSSION}

The oxygen consumption pattern during lipoprotein oxidation induced by AAPH was essentially similar to those for liposomal membranes (27) and erythrocyte membranes (15, 17). During the first phase of slow oxygen consumption, lipoprotein tocopherol was progressively consumed, indicating that tocopherol may have competitively inhibited the free radical chain oxidation of lipoprotein lipids by trapping radicals generated by AAPH. The finding that $\alpha$-tocopherol was consumed earlier than $\gamma$-tocopherol was similar to that of a previous study performed in homogenous solution (26). After the tocopherol concentrations fell below critical levels, the second phase of faster oxygen consumption commenced, indicating that extensive propagation of chain 
oxidative reactions was proceeding. Moreover, we found good correlations between $T_{\text {inh }}$ and tocopherol content, between $R_{\text {inh }}$ and the ratio of active $\mathrm{H}$ number to tocopherol content, and between $R_{\text {prop }}$ and active $H$ number. These findings are consistent with previous studies concerning the inhibition of lipid peroxidation by tocopherol in homogenous solution or in liposomal membranes performed by Niki et al. $(25,27,28)$. Our results also suggest that tocopherol can move around easily in the lipid portions of lipoproteins to act as a chain-breaking antioxidant effectively as it did in the case of above models. It was also shown that the oxidation of lipoprotein lipids could proceed by a free radical chain mechanism.

A comparison of the oxidation of neonatal and adult lipoproteins shows that $T_{i n h}$ was shorter and $R_{i n h}$ was larger in neonates. The shorter $T_{\text {inh }}$ is probably attributable to the lower tocopherol content in neonatal lipoproteins (Table 3 ), whereas the larger $\boldsymbol{R}_{\text {inh }}$ may have been due to the higher ratio of active $H$ to tocopherol (Table 4). This indicates the possibility that in newborn infants protection against lipid peroxidation of lipoproteins during periods of oxidative stress is insufficient because of the greater ratio of active $\mathrm{H}$ to tocopherol content in neonates. Although both neonatal active $\mathrm{H}$ numbers and tocopherol content are reduced compared with adult values, the decrease of tocopherol is more significant than that of active $H$ numbers. This explains the increased ratio of active $H$ to tocopherol content in neonates. After tocopherol depletion, $R_{\text {prop }}$ was greater in adult lipoproteins. This may have been due to the larger active $\mathrm{H}$ numbers in adult than in neonatal lipoproteins (Table 5). Under physiologic and even under pathologic conditions, such intensive oxidation of lipoproteins that lipoprotein tocopherol falls below critical levels is unlikely to occur. Therefore, it still seems reasonable to state that neonatal lipoproteins may be highly susceptible to oxidative stress, as indicated by their shorter $T_{\text {inh }}$ and larger $R_{\text {inh. }}$. Previously, Yoshioka et al. (29) reported that blood levels of thiobarbituric acid reactive substances (an index of lipid peroxides) were higher in newborn rats than in older rats. These findings agree with our results obtained from kinetic studies of lipoprotein oxidation with AAPH.

In the neonatal period, there is a great possibility of plasma lipoproteins being exposed to oxygen radicals under highly oxidative conditions, including hyperoxia induced by mechanical ventilation as in the case of respiratory distress in premature infants, asphyxia and the ensuing oxygen inhalation, polymorphonuclear cell activation and platelet aggregation induced by inflammation, or hemorrhage. Recently, Pitkänen et al. (30) reported that by quantitating ethane and pentane in expired air (an index of lipid peroxides) of very low birth weight infants with respiratory distress syndrome, infants in whom bronchopulmonary dysplasia later developed exhaled significantly more of these volatile hydrocarbons than did those with no serious neonatal complications. Wispe et al. (31) also reported that infants, including term healthy babies, expired higher levels of ethane and pentane compared with adults, and infants receiving parenteral lipid infusion excreted much higher levels of these volatile hydrocarbons. Although indirect measurements, these data would lead us to predict that lipid peroxidation practically may take place in vivo in newborn infants. Saugstad (32) emphasized the importance of hypoxanthine as an indicator of neonatal asphyxia and suggested the possibility that increased hypoxanthine may be a potential oxygen-free radical generator in neonates.

If the neonatal lipoproteins were exposed to oxidative stress under the conditions mentioned above, a rise in the tocopherol concentration of neonatal lipoproteins could cause the prolongation of $T_{i n h}$ as well as the reduction of $R_{i n h}$, and the peroxidizability of neonatal lipoproteins would be expected to be comparable to that of adult lipoproteins.

Our study suggested that compared with adults, newborn infants may be in a VE-deficient state as far as the protection of plasma lipids against oxidative stress is concerned, although red blood cell tocopherol concentrations are comparable to levels in adults and the plasma tocopherol/lipid ratio is above the critical level. Many investigations are presently being carried out to elucidate the contribution of oxidatively modified lipoproteins or lipid peroxidation products, such as lipid hydroperoxides and aldehydes, to various disorders. Recently, Lindeman et al. (33) reported that the total radical trapping capacity of the antioxidants in plasma was higher in the newborn babies than in adults. Our results concerning lipoprotein peroxidizability were derived from the examination of isolated lipoproteins. It is possible that a highly efficient plasma free radical trapping capacity at birth may partly compensate for the increased susceptibility of neonatal lipoproteins to oxidative stress. Thus, whether the susceptibility of neonatal lipoproteins to oxidative stress is really higher in vivo is open to question. Further studies are needed before the clinical consequences of our findings can be made clear.

Acknowledgments. The authors thank Drs. T. Uemura, $\mathrm{H}$. Tanaka, and T. Motoyama for kindly providing cord blood samples, and Eisai Co., Ltd. for providing soft capsule preparation of $\mathrm{d}$ - $\alpha$-tocopherol. We are also grateful to Dr. E. Niki, Professor of Tokyo University, for his kind help and discussion, and to $\mathrm{Y}$. Tabei and $\mathrm{M}$. Imanishi for their laboratory assistance. Thanks are also extended to D. Kaempf for her helpful assistance in the preparation of the manuscript.

\section{REFERENCES}

1. Steinberg D 1986 Studies on the mechanism of action of probucol. Am J Cardiol 57:16H-21H

2. Steinbrecher UP 1987 Oxidation of human low density lipoprotein results in derivatization of lysine residues of apolipoprotein B by lipid peroxide decomposition products. J Biol Chem 262:3603-3608

3. Heinecke JW 1987 Free radical modification of low-density lipoprotein: mechanism and biological consequences. Free Radic Biol Med 3:65-73

4. Esterbauer H, Jürgens G, Quehenberger O, Koller E 1987 Autoxidation of human low density lipoprotein: loss of polyunsaturated fatty acids and vitamin $E$ and generation of aldehydes. $J$ Lipid Res 28:495-509

5. Niki E, Komuro E 1989 Inhibition of peroxidation of membranes. In: Simic MG, Taylor KA, Ward JF, Sonntag C (eds) Oxygen Radicals in Biology and Medicine. Plenum Press, New York, pp 561-566

6. Ogihara T, Miki M, Kitagawa M, Mino M 1988 Distribution of tocopherol among human plasma lipoproteins. Clin Chim Acta 174:299-306

7. Horwitt MK, Harvey CC, Dahm Jr CC, Seary MT 1972 Relationship between tocopherol and serum lipid levels for determination of nutritional adequacy. Ann NY Acad Sci 203:233-236

8. Rubinstein HM, Dietz AA, Srinavasan R 1969 Relation of vitamin E and serum lipids. Clin Chim Acta 23:1-6

9. Martinez PE, Concalves AL, Jorge SM, Desai ID 1981 Vitamin E, placental blood and its relationship to maternal and newborn level of vitamin $E$. $J$ Pediatr 99:298-300

10. Mino M, Kitagawa M, Nakagawa S 1985 Red blood cell tocopherol concentrations in a population of Japanese children and premature infants in relation to the assessment of vitamin E status. Am J Clin Nutr 41:631-638

11. Tanaka H, Mino M, Takeuchi T 1988 A nutritional evaluation of vitamin E status in very low birth weight infants with respect to changes in plasma and red blood cell tocopherol leveis. J Nutr Sci Vitaminol (Tokyo) 34:293-307

12. Kitagawa M, Nakagawa S, Mino M 1983 Influence of plasma lipids and adiposity on red blood cell tocopherol level. Eur J Pediatr 140:238-243

13. Mino M, Nakagawa S, Tamai H, Miki M 1982 Clinical evaluation of red blood cell tocopherol. Ann NY Acad Sci 393:175-178

14. Lo SS, Frank D, Hitzig WH 1973 Vitamin E and haemolytic anaemia in premature infants. Arch Dis Child 48:360-365

15. Mino M, Miki M, Miyake M, Ogihara T 1989 Nutritional assessment of vitamin $E$ in oxidative stress. Ann NY Acad Sci 570:296-310

16. Hatch FT, Lees RS 1968 Practical methods for plasma lipoprotein analysis. Adv Lipid Res 6:1-68

17. Yamamoto Y, Niki E, Eguchi J, Kamiya Y, Shimasaki H 1985 Oxidation of biological membranes and its inhibition. Free radical chain oxidation of erythrocyte ghost membranes by oxygen. Biochim Biophys Acta 819:29-36

18. Shimasaki H, Philips FC, Privett OS 1977 Direct transesterification of lipids in mammalian tissue for fatty acid analysis via dehydration with $2,2^{\prime}$. dimethoxypropane. J Lipid Res 18:540-543

19. Miki M, Tamai H, Mino M, Yamamoto Y, Niki E 1987 Free-radical chain oxidation of rat red blood cells by molecular oxygen and its inhibition by $\alpha$ tocopherol. Arch Biochem Biophys 258:373-380

20. Allain CC, Poon LS, Chan CS, Richmond W, Fu PS 1974 Enzymatic determination of total serum cholesterol. Clin Chem 20:470-475

21. Takayama M, Itoh S, Nagasaki S, Tanimizu I 1977 A new enzymatic method for determination of serum choline-containing phospholipids. Clin Chim Acta 79:93-98

22. Bucolo G, David H 1973 Quantitative determination of serum triglyceride by the use of enzymes. Clin Chem 19:476-482 
23. Ishibashi K, Abe K, Ohmae M, Kawabe K, Katsui G 1977 Determination of tocopherols in red blood cells by high-performance liquid chromatography. [in Japanese] Vitamins 51:415-422

24. Tamai H, Manago M, Yokota K, Kitagawa M, Mino M 1988 Determination of $\alpha$-tocopherol in buccal mucosal cells using an electrochemical detector. Int J Vitam Nutr Res 58:202-207

25. Yamamoto Y, Niki E, Kamiya Y, Shimasaki H 1984 Oxidation of phosphatidylcholines in homogenous solution and in water dispersion. Biochim Biophys Acta 795:332-340

26. Niki E, Tsuchiya J, Yoshikawa Y, Yamamoto Y, Kamiya Y 1986 Oxidation of lipids. XIII. Antioxidant activities of $\alpha-, \beta-, \gamma-$, and $\delta$-tocopherols. Bull Chem Soc Jpn 59:497-501

27. Niki E, Takahashi M, Komuro E 1986 Antioxidant activity of vitamin E in liposomal membranes. Chem Lett 1573-1576

28. Niki E, Saito T, Kawakami A, Kamiya Y 1984 Inhibition of oxidation of methyl linoleate in solution by vitamin $\mathrm{E}$ and vitamin $\mathrm{C}$. $\mathrm{J}$ Biol Chem 259:4177-4182

29. Yoshioka T, Takehara Y, Shimatani M, Abe K, Utsumi K 1982 Lipid peroxidation and antioxidants in rat liver during development. Tohoku $\mathrm{J}$ Exp Med 137:391-400

30. Pitkänen OM, Hallman M, Andersson SM 1990 Correlation of free oxygen radical-induced lipid peroxidation with outcome in very low birth weight infants. J Pediatr 116:760-764

31. Wispe JR, Bell EF, Roberts RJ 1985 Assessment of lipid peroxidation in newborn infants and rabbits by measurements of expired ethane and pentane: influence of parenteral lipid infusion. Pediatr Res 19:374-379

32. Saugstad OD 1988 Hypoxanthine as an indicator of hypoxia: its role in health and disease through free radical production. Pediatr Res 23:143-150

33. Lindeman JHN, Van Zoeren-Grobben D, Schrijver J, Speek AJ, Poorthuis BJHM, Berger HM 1989 The total free radical trapping ability of cord blood plasma in preterm and term babies. Pediatr Res 26:20-24 